\title{
La homoparentalidad en la deconstrucción y reconstrucción de familia. Aportes para la discusión
}

Marcelo Robaldo'

\begin{abstract}
Resumen
El presente artículo aborda la contingencia desde la temática de la maternidad y paternidad homosexual. La discusión se enmarca dentro de una perspectiva de género y de un concepto de familia entendida como una arena de disputa de distintas prácticas y significados, donde las familias de homosexuales y lesbianas representan un desafío a los modelos tradicionales de parentesco. Se plantea por ende la necesidad de trascender los límites heteronormativos de los modelos del género en América Latina con el fin de visibilizar a dichas familias en la investigación social. Para esto se plantea la noción de homoparentalidad entendida como performatividad de los vínculos parentales en las familias no heterosexuales. A fin de comenzar a visualizar la especificidad de la problemática en nuestro país se comentan los hallazgos de una investigación comparada entre familias de lesbianas en Santiago y Barcelona. Se avanza de manera preliminar sobre algunos elementos de la problemática de los hombres homosexuales y la paternidad.
\end{abstract}

Palabras clave: homoparentalidad - género - familia - madres lesbianas - padres homosexuales.

\begin{abstract}
This article deals with the current day issue of homosexual mothering and fathering. Its arguments are set within a gender perspective and a concept of family as a site of contesting practices and meanings, in which homosexual families challenge traditional models of kinship. Thus, the article argues for the need to go beyond the hetero-normative boundaries of Latin-American gender theory in order to make these families visible to social research. In so doing it points to the idea of homosexual kinship as performativeness of parental bonds in non heterosexual families. Additionally, in seeking to visualize the specificity of the issue in Chile, comments are made on research findings from a comparative study carried out between Santiago and Barcelona. Finally, some preliminary ideas regarding the issue of homosexual men and fathering are also treated.
\end{abstract}

Key words: homosexual kinship - gender - family - lesbian mothers - gay fathers.

Sociólogo por la Universidad de Chile. Candidato a Doctor en Estudios de Género en la London School of Economics and Political Sciences, Inglaterra. Docente del Diplomado de Masculinidades y Políticas Públicas del Departamento de Sociología de la Universidad de Chile y del Magíster en Género y Cultura de la Facultad de Filosofía y Humanidades de la misma universidad. Participa en el Núcleo de Investigación en Género y Sociedad Julieta Kirkwood. 


\section{INTRODUCCIÓN}

Cuando vemos actualmente cómo se hace referencia a la familia dentro de una gran variedad de ámbitos, desde las políticas de Estado enfocadas en su promoción y apoyo hasta las publicaciones periódicas de la Iglesia católica declarando idéntico fin, surge naturalmente la pregunta acerca de qué distingue una u otra visión.

Sin duda, la familia se ha convertido en arena de disputa simbólica, ideológica e incluso política, donde compiten por legitimidad distintos proyectos de sociedad que muchas veces resultan contrapuestos.

Que la familia en tanto institución social es el sitio por excelencia donde se reproduce y literalmente se hace carne el género, no es novedad para la perspectiva de las ciencias sociales o de la teoría del género. Sin embargo, está fuera de los límites y pretensiones de este trabajo abordar las diversas aproximaciones teóricas que reviste el tema. Simplemente asumiremos su relevancia de hecho, para situarnos en la perspectiva en que la familia, en tanto institución social, se corresponde con la cultura y la historia y que, por ende, está siempre en transformación (Goody, 1986).

El hecho incontrovertible de este cambio puede constatarse hoy cuando se habla de una pluralidad de formas familiares. Las investigaciones sociales y los índices estadísticos, entre otros, hacen referencia a familias uni-personales, mono-parentales y bi-parentales, por mencionar sólo algunas. Sin embargo, en este discurso "oficial", que es culturalmente hegemónico y como tal comporta las leyes vigentes, las familias homosexuales no existen. El caso de la jueza Karen Atala es el ejemplo más claro².

La subvaloración de la homosexualidad en nuestra sociedad se sustenta dentro de un orden moral establecido desde el discurso del patriarcalismo y dice relación con la irrefutable condición de subalternidad que ocupan ciertos seres humanos dentro del entramado de relaciones de poder que se despliegan social y culturalmente en su interior, como han dado cuenta distintas autoras feministas (Spivak, 1998).

El trabajo de investigar la homoparentalidad tiene, por supuesto, consecuencias más allá del tema en sí. Es una problemática que conduce a replantear tanto los supuestos de la teoría acerca del parentesco como de la familia en general. El primer y principal hecho que las familias homosexuales vienen a subvertir es la naturalización del parentesco como un orden que se funda en la heterosexualidad. Judith Butler hace

En Chile, el caso de la jueza Atala se ha convertido en un ejemplo emblemático de la discriminación contra las madres lesbianas. A Karen Atala le es revocada la tuición de sus tres hijas en mayo de 2004, tuición que se otorga a su ex esposo y padre de las niñas, en circunstancias que no existe antecedente alguno que amerite no darle la tuición sobre sus hijas a la madre, dejando de manifiesto que es su opción sexual lésbica, vivida abiertamente, lo que determinó el fallo de la Corte Suprema en su contra. 
explícito este quiebre al preguntarse si acaso el parentesco es siempre y por defecto heterosexual, abriendo la discusión sobre la manera en que la teoría social ha entendido tradicionalmente el parentesco. La autora postula un concepto de parentesco cercano a sus ideas del género como performatividad y sostiene que el parentesco se hace, que es sujeto de transformaciones. Por cierto nos detendremos sobre este punto más adelante.

Que el parentesco no necesariamente se funde en lo heterosexual también tiene implicancias para nuestras ideas acerca del género. Tanto la antropología del parentesco, en sus primeros momentos, como el psicoanálisis freudiano e incluso el lacaniano están circunscritos dentro de lo que podemos denominar paradigma heteronormativo $^{3}$. Sin embargo, la existencia de familias homosexuales pone en duda algunos de los supuestos más fundamentales que el sentido común y las teorías científicas abrigan sobre la sexualidad y el género. Se entiende comúnmente que la identidad de género se funda en la diferenciación complementaria que hay entre los sexos, con una hembra y un macho, dispuestos biológicamente para dar origen a un nuevo ser. Las nociones del tabú del incesto y el proceso de edipalización, tal como los entiende el psicoanálisis, justamente se orientan en la dirección de estos supuestos del género como una complementariedad de opuestos.

¿Qué pasa, sin embargo, cuando ya no es la procreación lo que funda el parentesco? ¿Cómo varían los procesos que llevan a los hijos de familias homosexuales hacia la constitución de la identidad de género? ¿Qué maneras de "hacer"familia hay fundadas fuera de la norma heterosexual, fuera de la heteronormatividad? Butler plantea estas preguntas y sugiere que la homoparentalidad implica un sentido radicalmente distinto del parentesco, asociándolo al género, pues concibe a ambos como un hacer, como performatividad.

\section{LA INVESTIGACIÓN EN HOMOPARENTALIDAD DESDE LA PERSPECTIVA DE LA EPISTEMOLOGÍA DEL GÉNERO}

Ciertamente, las implicancias de estudiar la homoparentalidad también radican en lo epistemológico. Algunas epistemólogas del género (Harding, 2004; Hartsock, 1983) han afirmado que el sujeto subalterno guarda un privilegio epistemológico respecto de aquel que ejerce la dominación: se trata de la teoría del standpoint ${ }^{4}$. Evidentemente, esta idea no es exclusiva de la epistemología feminista, pero en ella encuentra una expresión particular toda vez que, planteada desde aquí, los sujetos aludidos encarnan un género, son hombres o mujeres y precisamente estas últimas serían quienes gozan del privilegio del que hace mención la epistemología del standpoint.

Al respecto, véase Rich (1980).

Sobre la epistemología de standpoint, véase Harding (2004). 
Si bien las mujeres, por su ubicación en la reproducción de la vida, están en una posición privilegiada para observar las contradicciones del orden masculinista, son bases de este orden masculinista tanto la homofobia como la misoginia. Tienen entonces las mujeres lesbianas y los hombres homosexuales una experiencia privilegiada también para develar las formas en que el patriarcalismo despliega su poder.

Algunas epistemólogas feministas, como Donna Haraway (1988), sostienen que el privilegio epistemológico se da desde cierta ubicación o lugar dentro de las relaciones de género y, en este sentido, abre la discusión sobre si la condición de mujer a secas es suficiente para otorgar tal privilegio. Más bien, la posibilidad de tal epistemología, aquella que no sea cómplice de una mirada "neutra" que mira todo desde ninguna parte (aquello que las epistemólogas feministas anglosajonas llaman el "god trick"), se encuentra en explicitar el lugar del observador e incorporarlo dentro de las consideraciones del proceso de producción de conocimientos, es decir, no sacar al observador de la "realidad" estudiada, como ha sido tradicionalmente la pretensión del paradigma objetivista de las ciencias.

En relación a esto último, vemos que en Chile la investigación en paternidad no ha logrado situarse fuera de sus límites heteronormativos, al excluir de su producción de conocimiento a los padres homosexuales. El hecho es que en Chile hay casos de padres homosexuales que han reclamado con éxito la tuición de sus hijos y, sin embargo, la investigación sobre masculinidad y paternidad no les presta ni les ha prestado atención.

Convengamos en que el hacer familia para las parejas homosexuales se logra desde una matriz distinta a la heterosexual y fuera de la heteronormatividad. Es más, esta heteronormatividad rige epistemológicamente sobre cierta perspectiva de los estudios de género en América Latina, aquella que postula la ausencia del padre como el elemento histórico y cultural gravitante en la matriz de las relaciones de género, caracterizada por la díada "huacho-madre" (Salazar, 1990).

Este planteamiento está inscrito dentro de lo que llamaremos epistemología heteronormativa. Como tal, no trasciende una noción del parentesco fundado en la procreación y mantiene a pie firme la idea de que el individuo ingresa a la cultura y adquiere una identidad de género a partir de la complementariedad de los opuestos que implica la heterosexualidad, en tanto división tajante entre lo femenino y lo masculino. En efecto, frente a la pregunta por la homoparentalidad, tanto la Historia como la Antropología del género en Chile no han demostrado mayor preocupación, permaneciendo circunscritas dentro de un paradigma del parentesco estrictamente heterosexual.

Como veremos más adelante, muchas veces las parejas homosexuales buscan construir sentidos de familia más allá de lo que se ha llamado la regla de los padres progenitores (Cadoret, 2003). En el contexto de familias homosexuales en Chile, parti- 
cularmente en el caso de los varones, no sabemos cuánto es lo que realmente pesa la sangre al momento de hacer familias.

\section{LA HOMOPARENTALIDAD: HOMOSEXUALIDAD, PARENTESCO Y EL ORDEN SIMBÓLICO}

Para Judith Butler (2006) el matrimonio homosexual no es lo mismo que el parentesco homosexual, sin embargo, éstos se han confundido. Según la autora, en la opinión pública estadounidense el matrimonio debiera continuar siendo una institución y un vínculo heterosexual y, por tanto, las uniones gays no son relaciones de parentesco. Así, según esta visión, la sexualidad necesita organizarse al servicio de las relaciones reproductivas y el matrimonio debiera permanecer como el punto de apoyo que mantiene en equilibrio a las instituciones de la familia y el parentesco, las que existen una en virtud de la otra.

Esta noción del matrimonio enfrenta, tanto en Estados Unidos como en muchas otras sociedades, los desafíos que plantea la realidad sociológica en la que existe cierta cantidad de relaciones de parentesco que no se conforman según el modelo de la familia nuclear y reproductiva y que, por tanto, exceden el alcance de las actuales concepciones jurídicas, operando según reglas que no se pueden formalizar (Ibíd.).

Según Butler, la sociología y antropología recientes muestran cómo las nociones de parentesco se han desvinculado de la presunción del matrimonio. Así, por ejemplo, el antropólogo chino Cai Hua refuta la visión del parentesco de Lévi-Strauss como una negociación de la línea patrilineal a través de los lazos del matrimonio. Entre los na, etnia china, ni los maridos ni los padres juegan un papel prominente en la determinación del parentesco.

En consecuencia, la autora plantea una definición de parentesco más amplia y señala que éste implica:

"una serie de prácticas que instituyen relaciones de varios tipos mediante las cuales se negocian la reproducción de la vida y las demandas de la muerte (...) las prácticas de parentesco serán aquellas que surjan para cuidar de las formas fundamentales de la dependencia humana, que pueden incluir el nacimiento, la crianza de los niños y las relaciones de dependencia emocional y de apoyo [entre otras]" (Ibíd: 150).

Anne Cadoret (Op. Cit.) señala que las parejas homosexuales en Francia intentan dar coherencia a una identidad familiar atendiendo a dos cuestiones: la importancia de lo biológico como fundamento de la familia, por un lado, y la adecuación entre pareja parental y pareja conyugal, por otro. Es respecto a los elementos de este segundo punto que se asocian los debates en torno a la homoparentalidad y matrimonio homosexual, respectivamente. 
La homoparentalidad comporta distintos arreglos de reproducción y cuidados. Están las parejas que crían a sus hijos en base a un modelo de co-parentalidad, por ejemplo, las parejas lesbianas que comparten la tarea de criar a los niños con una pareja de hombres homosexuales o con un donante conocido, buscando mantener un modelo basado en la regla padres-progenitores (Ibíd.). Este también es el caso de las familias por inseminación artificial (IA). En cambio, para las familias adoptivas, donde no es posible mantener la regla padres-progenitores, el modelo se asienta en un parentesco social (en vez de biológico).

Si bien es cierto que las familias por IA refuerzan el valor simbólico de lo biológico en el establecimiento del parentesco, mientras que las familias adoptivas lo hacen a través del parentesco social, Cadoret señala que las familias homosexuales, en todas sus formas, añaden un nuevo distanciamiento del modelo familiar de referencia (sea éste con énfasis en lo biológico o en lo social) y una apertura hacia el multi-parentesco.

\section{FAMILIAS LÉSBICAS. PROBLEMÁTICAS ACERCA DE LA MATERNIDAD}

En su investigación comparativa entre familias de lesbianas asentadas en las ciudades de Barcelona y Santiago de Chile, Florencia Herrera (2005) señala que las primeras habitan en un contexto donde se reivindica el reconocimiento de las formas alternativas de familias, como pueden serlo justamente aquellas conformadas por parejas lésbicas. Así, más que un énfasis en la diferenciación o en el rechazo de los modelos de familia existentes, hay una exigencia de legitimación legal y social de las familias que ellas forman. No hay, por lo tanto, una definición de familia homosexual en oposición a la de familia heterosexual. Las mujeres entrevistadas por Herrera no observan grandes diferencias entre las familias que ellas, como lesbianas, han construido y lo que se podría llamar el modelo de familia heterosexual. Es decir, las narrativas de las lesbianas hablan de inclusión más que de diferenciación.

Por otro lado, este estudio revela que entre las parejas de lesbianas chilenas poder conciliar la maternidad y la homosexualidad constituye un dilema, pese a su anhelo de ser reconocidas como familias legítimas. Así, para las lesbianas que son madres producto de relaciones previas y heterosexuales, no es lícito plantearse una vida en pareja homosexual. Ello transgrede justamente la regla de los padres-progenitores.

La aprensión de estas parejas es provocar un daño a los/as hijos/as. Cadoret (Op. cit.), en este sentido, señala que el origen de este temor está en la estructura diádica y heterosexual de la parentalidad, dentro de la cual se estima que un niño o niña debe tener un padre y una madre para contar con una infancia adecuada, puesto que para ingresar a la cultura los/as niños/as necesitan un origen en la diferencia sexual, cuestión que ha señalado Lacan (1977) como propia del orden simbólico y que, como tal, es permanente. 
De alguna forma, el modelo psicoanalítico del deseo no da cuenta de las familias homosexuales en tanto no atiende el hecho de la subvaloración de la homosexualidad en la cultura patriarcal. Si bien para Lacan lo importante es el proceso de edipalización a nivel del orden simbólico, la sub-valorización de lo femenino y lo homosexual hace que en la discusión acerca de las familias lésbicas, donde los términos y las posiciones de padre y madre del triángulo edípico son ocupados por mujeres exclusivamente, sea necesario introducir una perspectiva de política sexual.

En consecuencia, resulta relevante plantear que, aun cuando el psicoanálisis lacaniano plantea que son las posiciones en el triángulo del deseo edípico más que la mujer o el hombre concreto que esté ocupándolas lo relevante, el hecho de que una lesbiana, es decir, una mujer no heterosexual, ocupe la posición del patriarca pone de cabeza a este paradigma. El punto es que dicho paradigma no llega a cuestionar lo que el orden simbólico tiene de heteronormativo, vale decir, no da cuenta de cómo los fundamentos de las familias lesbianas, lo femenino y lo homosexual niegan el supuesto requisito central del orden simbólico para el génesis de la identidad sexual, como es el origen del individuo en la diferencia sexual.

Cabe preguntarse, haciendo eco de Butler, ¿cómo podemos empezar a comprender qué formas de diferenciación de género tienen lugar en el niño cuando la edipalización no presupone la heterosexualidad?

En efecto, para la antropología estructural de Lévi-Strauss (1969), es su entrada en el orden simbólico la que constituye al sujeto. No hay sujeto posible fuera del orden simbólico, es decir, no hay sujeto posible fuera del lenguaje y del origen en la diferencia sexual. Por cierto, en el sistema de sexo-género opera la heterosexualidad obligatoria como norma dada desde el orden simbólico, sin embargo, como ya lo hemos indicado, aquello está en transformación.

Según Judith Butler (2006), este postulado de una heterosexualidad fundadora debe también ser leído como parte de la operación del poder y de la fantasía, a mayor escala. Así, para la autora, es válido preguntar cómo funciona el cambio de dicho fundamento en la construcción del Estado y la nación.

En el debate francés sobre las PACS ("Pactos de Solidaridad Civil", que constituyen una alternativa al matrimonio para cualquier pareja de individuos, independientemente de su orientación sexual), la discusión sobre el matrimonio o parentesco gay, dos cuestiones que, como hemos dicho, a menudo se fusionan, se ha convertido en un espacio de desplazamiento de otros miedos políticos: miedos sobre la tecnología, la nueva demografía, la propia unidad de la nación y miedo a que el feminismo, al insistir sobre el cuidado de los/as niños/as, haya, de hecho, colocado al parentesco fuera de la familia incorporando a extraños. 
En este sentido, la misma autora ha llamado la atención sobre las afirmaciones de algunas filósofas francesas conservadoras relativas a que la maternidad y/o paternidad homosexual violenta el orden simbólico. Según Eric Fassin (s.f.; citado en Butler, 2006), estas afirmaciones sobre el orden simbólico, que asocian necesariamente el matrimonio a la filiación, deben ser entendidas como una respuesta compensatoria a la ruptura histórica del matrimonio como institución hegemónica.

Para David Schneider (s.f.; citado en Ibíd.), el parentesco es una especie de hacer que no refleja una estructura anterior. Lejos de suponer una heterosexualidad fundante, que pone como condición de ingreso a la cultura el tabú del incesto y el proceso de edipalización como mecanismos diferenciadores de género, reproduciendo la heterosexualidad normativa y la identidad de género diádica y diferenciada, el parentesco sólo puede entenderse como una práctica representada, como un hacer.

En relación a lo anterior, Butler (Ibíd.) señala que esta idea

"(...) nos permitiría evitar que una estructura de relaciones hipostatizada se oculte detrás de los actuales acuerdos sociales y nos permitiría considerar el cómo los modos de hacer, pautados y performados, hacen funcionar a las categorías de parentesco y se convierten en los medios a través de los cuales las categorías experimentan una transformación y/o desplazamiento" (178).

Resulta relevante destacar cómo el uso de la noción de perfomatividad se extiende aquí más allá de su origen en la identidad de género para explicar también el parentesco y, tal como en el caso de la performatividad de género, la idea de que el parentesco se "hace" nos ofrece una salida a las prácticas hegemónicas.

En el entendido de lo anterior, es pertinente la investigación de Herrera (2005) respecto a los modos de hacer, pautados y performados, de la maternidad lésbica. La autora, siguiendo a Jeffrey Weeks, sostiene que"las relaciones homosexuales cuestionan los elementos centrales en la comprensión tradicional de la familia: la diferencia de sexo de la pareja con respecto a la afinidad y la consanguinidad en relación con la filiación" (Ibíd: 270). Aunque Herrera no enfoca su trabajo desde la crítica feminista o la teoría de género, entiende que "las prácticas y narrativas que las mujeres homosexuales construyen a partir de sus relaciones más cercanas son de gran utilidad a la hora de comprender las transformaciones que las formas de hacer familia están sufriendo en nuestros tiempos" (Ibíd: 270-271). Agrega que, si bien es dudoso generalizar al hablar de homosexualidad, existe un denominador común en la vida de todo homosexual: el estigma y la injuria, denominadores comunes que los llevan a recorrer lo que Erving Goffman (1998; citado en Ibíd.) Ilama 'carreras morales' similares.

En la misma investigación, tanto lesbianas catalanas como chilenas se oponen a la afirmación de que por tener sexo no procreativo son infértiles. Con esto, la idea de que los homosexuales no pueden procrear es refutada, y así como en algún momento se 
separó sexualidad de reproducción, ahora se separa la orientación sexual de un orden familiar determinado.

Las lesbianas chilenas, sin embargo, tienen una relación conflictiva con la maternidad. Muchas de ellas han sido madres a raíz de una relación heterosexual anterior. Así, les es más difícil vincular la maternidad con una identidad homosexual. Para las lesbianas españolas, en cambio, el dilema en relación a su parentalidad y crianza lo representa la legalidad de su maternidad, es decir, el no reconocimiento como madres legítimas. Al respecto, Herrera (Ibíd.) señala que "en el contexto catalán, para la mayoría de las lesbianas la combinación de la maternidad con su identidad sexual no presenta ningún dilema moral. Más bien, existe la idea generalizada de que una pareja homosexual puede ser tan buena en la crianza de niños como una pareja heterosexual" (273-274).

En este sentido, tanto en Barcelona como en Santiago, los problemas asociados a la maternidad lésbica se perciben como externos a la pareja homosexual, es decir, los principales obstáculos que enfrenta una pareja lésbica que tiene o quiere tener hijos son de orden social, por el no reconocimiento y la discriminación por parte de la sociedad de sus relaciones íntimas.

Aunque, en efecto, las lesbianas chilenas no cuestionan directamente la capacidad de crianza de una pareja de mujeres, sí ven con preocupación el futuro del niño o niña. Esta inquietud está relacionada con la discriminación y estigmatización que creen sufriría un/a niño/a criado/a por madres lesbianas. Es tan grande esta preocupación que, por lo general, las madres lesbianas chilenas sostienen que jamás vivirán en pareja con otra mujer mientras sus hijos e hijas aún vivan con ellas (Ibíd: 274). El temor es provocarles un trauma, inducido por darse cuenta que tener dos madres no es normal ni bien visto por la sociedad en la que viven.

Entre las lesbianas de Barcelona, los problemas no se refieren a las relaciones internas de la familia homosexual, más bien están en las dificultades técnicas y prácticas que enfrenta una lesbiana o una pareja lésbica a la hora de decidir tener un hijo. Estos son, según la autora: i) el no reconocimiento de la pareja y, por lo tanto, la negación de una maternidad compartida legalmente; ii) las dificultades que enfrenta una persona al querer adoptar y la imposibilidad de adoptar como pareja; iii) el no reconocimiento de la madre 'no legal', en el caso de tener un hijo en común; y iv) la falta de referentes de familias alternativas en las escuelas y en los medios de comunicación, entre otros.

\section{LA FAMILIA TECNOLÓGICA}

Volviendo al tema planteado por Butler respecto a los miedos sobre la tecnología que se han desplazado hacia el interior del debate sobre la familia homosexual, es relevante detenerse en la faceta tecnológica que también implican estas nuevas familias y, así, destacar el trabajo de Bohannan (1992; citado en Herrera, Op. cit.), quien plantea que se debe distinguir entre la procreación, que implica los procesos biológicos 
de concepción y parto, y la reproducción, que incluye a la primera además del trabajo de llevar la progenie a la edad adulta. Sí, este último concepto incluye tanto el acto sexual como el cuidado y enculturación, es decir, la crianza. La procreación se considera un hecho eminentemente biológico, mientras la reproducción incluye también ingredientes culturales y sociales.

De acuerdo con este autor, si la sexualidad está culturalmente circunscrita a la procreación, la homosexualidad será considerada anómala porque la procreación no puede tener lugar en un acto sexual de dos personas del mismo sexo. En efecto, según Herrera (Ibíd.), en Chile se utiliza el argumento de que los homosexuales no tienen capacidad procreativa para excluirlos del parentesco y así fundamentar la negación de sus derechos al matrimonio y la adopción.

Tales argumentos pierden legitimidad ante la existencia de lo que podríamos llamar la familia tecnológica. Ésta constituye el sitio de eclosión principal de los nuevos "experimentos de vida", como los han llamado (Weeks, 2001) aludiendo a las familias homosexuales donde los métodos de fertilidad asistida, como la inseminación artificial (IA), desligan "procreación" de "naturaleza".

Esto pone de manifiesto la distinción que hace Bohannan respecto a que la procreación es parte de la reproducción, colocándonos así frente a la cuestión de cómo la maternidad en sí es también un hecho del orden de la reproducción, toda vez que la procreación está crecientemente desligada de su representación común, como queda de manifiesto en lo dicho anteriormente acerca de las familias lésbicas. Y, dentro de las familias tecnológicas, son quizás las familias de homosexuales hombres las que en mayor medida transgreden el orden natural, puesto que al ser cuerpos masculinos y, como tal, no estar inscritos en el registro del cuerpo-útero, en menor medida aun que las lesbianas pueden ellos participar de la procreación.

\section{LA PATERNIDAD}

Aun cuando todo lo anterior, sobre cómo forman familias lesbianas catalanas y chilenas, representa un cambio radical en lo que se considera aceptable en nuestra sociedad, quizás lo que desafía más abiertamente el orden tradicional es que dentro de estas familias no exista un padre. Lo que esto subvierte es, como ya se ha señalado, la regla básica del parentesco, según la cual la paternidad y la maternidad deben organizarse en base a la diferencia de sexo y de roles que esta diferencia supone.

Si ya es un desafío para los modos preestablecidos de hacer la familia que las parejas lesbianas establezcan familias sin un padre, es quizás un desafío mayor para el orden "natural" el que exista una familia que no tenga madre. Ahora, Bohannan (citado en Herrera, Op. cit.), al aclarar que las tareas de reproducción que subyacen a la procreación no excluyen a las parejas homosexuales, abre las puertas a la posibilidad de que entre hombres también se formen familias. 
No existe en el medio chileno investigación acerca de la paternidad homosexual. De ahí que lo que se puede aseverar acerca de ésta es prácticamente nada. La investigación acerca de la paternidad en Chile no ha profundizado en el análisis en torno a la relación entre identidad sexual y significado de familia. Cabe hacer eco de las preguntas planteadas por Herrera y Butler en torno al tema: ¿cuán desafiante es para la teoría del parentesco y para nuestra sociedad el que las familias estén compuestas por parejas homosexuales? Y, ¿cuál es la fantasía del amor homosexual que el hijo inconscientemente adopta en las familias gay?

\section{CONCLUSIÓN}

Para una agenda de igualdad de género es relevante poner de manifiesto que el patriarcado subvalora lo femenino y lo homosexual en tanto términos de pasividad y receptividad. Es prioritario dar espacio a las familias homosexuales dentro de lo que se reconoce como legítimo, toda vez que al interior de éstas conviven personas con idénticos derechos al resto y que, sin embargo, en lo relativo a sus aspiraciones de "cuidar y ser cuidados", como escribe Herrera (Ibíd.), son ciudadanos, en el mejor de los casos, de segunda categoría.

Se requiere, entonces, problematizar la pregunta sobre cómo, además de la opresión de la mujer, está comprometida también la opresión de ciertas formas de hacer el género entre hombres, que desde luego son las formas no hegemónicas, siendo una de éstas la paternidad de hombres no heterosexuales.

En definitiva, el espacio de las familias homosexuales se entiende mejor como un ejercicio reflexivo, en el sentido que la sociología ha dado a este concepto dentro de su concepción de la modernidad. Además, es relevante visibilizar a las familias no heterosexuales desde un punto de vista sociológico porque, tal como indicamos en un comienzo, deconstruir los discursos de los ideologemas de familia tiene implicancias más allá del tema de las familias homosexuales. La homoparentalidad es una plataforma idónea para entrever las prácticas performativas del parentesco y el género, toda vez que en la raíz del prejuicio contra lo homosexual habita, como señala Butler, una prohibición del deseo anterior incluso al tabú del incesto. Indagar en los orígenes de tal prohibición es ir a las bases de la identidad de género y, en consecuencia, a lo que es primigenio en la constitución de lo humano.

\section{BIBLIOGRAFÍA}

Barret, Robert y Robinson, Bryan (2000): Gay fathers: encouraging the hearts of gay dads and their families. San Francisco: Jossey-Bass.

Benjamin, Jessica (1992): Los lazos de amor, psicoanálisis, feminismo y el problema de la dominación. Buenos Aires: Paidós. 
Butler, Judith (1999): Gender trouble feminism and the subversion of identity. Nueva York: Routledge.

--------- (2006): Deshacer el género. Barcelona: Ediciones Paidós Ibérica.

Cadoret, Anne (2003): Padres como los demás: homosexualidad y parentesco. Barcelona: Gedisa.

Flax, Jane (1995): Psicoanálisis y feminismo. Madrid: Editorial Cátedra.

Giddens, Anthony (1990): The consequences of modernity. Cambridge: Polity.

(1993): The transformation of intimacy: sexuality, love and eroticism in modern societies. Cambridge: Polity.

(1997): Modernidad e identidad del Yo. El YO y la sociedad en la época contemporánea. Barcelona: Península.

Goody, Jack (1986): La evolución de la familia y del matrimonio en Europa. Barcelona: Ed. Herder.

Gutmann, Matthew (2003): Changing men and masculinities in Latin America. Durham: Duke University Press.

Hartsock, Nancy (1983): "The feminist standpoint", en Hintikka y Harding (eds.): Discovering reality, pp. 283-310. Holland, Boston, London: D. Riedel Publishing Company.

Herrera, Florencia (2005): "Familia y maternidad: sangre y cuidado en mujeres lesbianas", en Valdés y Valdés (comp.): Conservadurismo y trasgresión en Chile: reflexiones sobre el mundo privado, colección Investigadores Jóvenes. Santiago: FLACSO / CEDEM.

Lacan, Jacques (1977): Écrits: a selection. Nueva York: Norton.

Laqueur, Thomas (1994): La construcción del sexo: cuerpo y género desde los griegos hasta Freud. Madrid: Cátedra.

Lévi-Strauss, Claude (1969): The elementary structures of kinship. Boston: Beacon.

Oyarzún, Kemy (2000): "La familia como ideologema. Género, globalización y cultura. Chile, 1989-1997", en Revista Chilena de Humanidades, No. 20, pp. 115-146. Santiago de Chile: Universidad de Chile, Facultad de Filosofía y Humanidades.

Rich, Adrienne (1980): "Compulsory heterosexuality and lesbian existence", en Signs: Journal of Women in Culture and Society, No. 5, pp. 631-60. 
Spivak, Gayatri (1998): "Can the subaltern speak?", en Nelson y Grossberg (comp.): Marxism and the Interpretation of culture. Chicago: University of Illinois Press.

Weeks, Jeffrey (2001): Same sex intimaces: families of choice and other life experiments. Londres: Routledge. 